\title{
Análisis del comportamiento a flexión de muros de adobe reforzados con geomallas
}

\section{Bending behavior analysis of geogrid reinforced adobe walls}

\author{
M. Solís ${ }^{(*)}$, D. Torrealva ${ }^{(*)}$, P. Santillán ${ }^{(* *)}$, G. Montoya ${ }^{(*)}$
}

\section{RESUMEN}

La tierra cruda constituye el material de construcción más importante en muchas regiones del planeta. Dado el riesgo sísmico existente en la mayor parte de estas regiones, es necesario el desarrollo de técnicas de refuerzo eficaces y adecuadas desde un punto de vista tecnológico y socioeconómico. Este artículo analiza desde un punto experimental y analítico el comportamiento a flexión de muros de adobe reforzados con geomallas. Las leyes momento-curvatura de los muros ensayados son aproximadas mediante una serie de modelos analíticos que permiten analizar el comportamiento del material compuesto adobe-geomalla. Los resultados obtenidos muestran como la geomalla mejora el comportamiento de la mampostería de adobe en términos de resistencia y ductilidad, mejorando así su comportamiento en caso de movimiento sísmico.

Palabras clave: Adobe; refuerzo con geomallas; diseño sísmico; comportamiento a flexión.

\section{ABSTRACT}

Rammed earth is a widely used building material in many regions of the world. Due to the high seismic risk in those areas, earthen constructions require suitable and efficient reinforcement techniques from a technological and socioeconomic point of view. This paper analyzes the bending behavior of geogrid reinforced adobe walls from an experimental and analytical point of view. The experimental bending moment-curvature relationships are analytically approached. The results show how geogrid reinforcement improves the performance of adobe masonry in terms of strength and ductility. Thus, a better seismic performance is achieved.

Keywords: Adobe; geogrid reinforcement; seismic design; bending behavior.

${ }^{(*)}$ ETS Ingeniería - Universidad de Sevilla (España).

${ }^{(* *)}$ Pontificia Universidad Católica del Perú (PUCP), Lima (Perú).

Persona de contacto/Corresponding author: msolis@us.es (M. Solís)

Cómo citar este artículo/Citation: Solís, M., Torrealva, D., Santillán, P., Montoya, G. (2015). Análisis del comportamiento a flexión de muros de adobe reforzados con geomallas. Informes de la Construcción, 67(539): eo92, doi: http://dx.doi.org/10.3989/ic.13.141.

Licencia / License: Salvo indicación contraria, todos los contenidos de la edición electrónica de Informes de la Construcción se distribuyen bajo una licencia de uso y distribución Creative Commons Reconocimiento no Comercial 3.o. España (cc-by-nc). 


\section{INTRODUCCIÓN}

La tierra es uno de los materiales de construcción más antiguos empleados por el hombre. Actualmente la construcción con tierra cruda (sin cocer) es considerada como una tecnología de bajo coste por la fácil disponibilidad de los materiales y la facilidad en su manejo, lo cual hace que actualmente sigan recurriendo a ella amplios sectores de población mundial Este artículo se centra en la mejora del comportamiento mecánico de este tipo de material, concretamente de la mampostería de adobe, por lo que de aquí en adelante al hablar de tierra como material de construcción deberá entenderse tierra cruda.

Actualmente se estima, según los trabajos de diversos autores (1) (2), que entre un 30 y un $50 \%$ de la población mundial vive en viviendas de tierra. Además de su bajo coste, la construcción en tierra ofrece excelentes propiedades en cuanto aislamiento (térmico y acústico) y sostenibilidad ambiental. Esto hace que sea creciente su uso en países desarrollados de todo el mundo (2) (3) (4) (5).

En el caso de Perú, país donde se ha llevado a cabo el trabajo presentado en este artículo, el $35 \%$ de las viviendas peruanas son de adobe y están habitadas por más de 9 millones de peruanos, según el censo oficial de 2007.

Por otro lado, aproximadamente un $10 \%$ del Patrimonio Mundial de la Unesco son construcciones de tierra, y éste constituye a su vez el $60 \%$ del considerado en peligro de destrucción o ruina (6) (7). Esto indica la relevancia y al mismo tiempo la vulnerabilidad de este tipo de construcciones. Algunos casos representativos se encuentran ubicados en Perú, tales como las ruinas de Chan Chan o algunos edificios del centro histórico de Lima.

Ante la importancia del empleo de la tierra cruda como material de construcción, y el hecho de que muchas de las áreas geográficas donde resulta habitual la construcción con tierra coincidan precisamente con zonas del planeta de elevada actividad sísmica, se hace necesario desarrollar métodos de análisis estructural y sistemas de refuerzo apropiados para conseguir niveles adecuados de seguridad frente al riesgo sísmico. Los esfuerzos encaminados a dar solución a esta problemática, no solo están justificados por simple justicia social, sino que también son necesarios para reducir los costes sociales y económicos que tiene para la comunidad internacional las situaciones catastróficas provocadas por movimientos sísmicos (8) (9).

Hasta el momento, existen significativos estudios científicos dedicados al estudio de las propiedades mecánicas y físicoquímicas del adobe, (10) (11) (12) (13) (14) pero son pocos los trabajos dedicados a su comportamiento estructural, reduciéndose la mayoría de ellos a estudios experimentales y análisis o principios de diseño cualitativos (15) (16) (17). Los trabajos dedicados a desarrollar modelos de cálculo son muy escasos, y se encuentran dedicados a aproximaciones numéricas del comportamiento global de alguna edificación particular, careciendo de estudios previos acerca del comportamiento estructural de muros aislados (18) (19) (20) (21). El número de trabajos dedicados al análisis del comportamiento de muros de adobe es limitado. Se han realizado ensayos experimentales de su comportamiento a cortante con y sin refuerzo con malla sintética (13) y también existen para este tipo de ensayos tentativas de modelado de acuerdo a modelos numéricos de simulación de mampostería (22). En cuanto al comportamiento a flexión de muros, el único trabajo previo conocido por los autores se refiere al análisis experimental de pequeños muretes de bloques de tierra comprimidos y estabilizados con cemento (23).

En las últimas décadas, se han estudiado diversas técnicas de refuerzo para mejorar las propiedades resistentes del adobe. En este sentido los trabajos desarrollados en la Pontificia Universidad Católica del Perú son una referencia internacional. En ellos se ha analizado cualitativamente el comportamiento sismorresistente a partir de ensayos de simulación sísmica sobre módulos de viviendas a escala real en una mesa vibradora. Así, se ha investigado el refuerzo con materiales naturales (paja, caña, madera, etc.) y también materiales industriales como mallas de alambre, parrillas de acero electrosoldadas, tubos de PVC y otros materiales plásticos. Un resumen de todos estos trabajos se puede encontrar en el artículo de Blondet et al. (24). Sin embargo, estos trabajos han aportado tan solo información cualitativa sobre el comportamiento de los refuerzos pero no han desarrollado modelos matemáticos que cuantifiquen y permitan realizar con rigor un análisis comparativo el efecto de cada sistema de refuerzo.

Dentro de estas propuestas de refuerzo, la que ha ofrecido unas mejores prestaciones consiste en el refuerzo de muros de adobe con un material polimérico (geomalla) (24) (25) (26). Con este refuerzo se consigue por un lado aumentar la resistencia de los muros de adobe, aumentando fundamentalmente su capacidad para soportar tensiones de tracción, pero más importante aún es el aumento de la ductilidad de la construcción, que permite la disipación de energía en caso de sismo. La geomalla además actúa como medio de confinamiento de la mampostería de adobe, de forma que reduce el riesgo de colapso de la estructura aun cuando se llegan a producir grandes desplazamientos en la misma.

Además de las ventajas desde un punto de vista estructural, la técnica de refuerzo propuesta ofrece la ventaja de poder ser aplicable sobre estructuras ya existentes, sin afectar a su apariencia externa. Además, ofrece buena durabilidad y resistencia a la corrosión, resulta asequible económicamente, y desde un punto de vista ingenieril, ofrece unas propiedades mecánicas certificadas por el fabricante que pueden servir para predecir de forma más segura y fiable su comportamiento junto con el adobe.

En este artículo se analiza el comportamiento a flexión de este material compuesto formado por adobe y refuerzo de geomalla, planteando modelos de comportamiento analíticos que permiten analizan matemáticamente las mejoras aportadas por el sistema de refuerzo. Para ello se presentarán en primer lugar los resultados de ensayos de flexión de muros con y sin refuerzo. Posteriormente se plantearán las ecuaciones que gobiernan el problema de flexión y se propondrán modelos analíticos basados en modelos constitutivos de adobe y geomalla para reproducir de forma aproximada los resultados experimentales en forma de leyes momento-curvatura. Los resultados obtenidos con distintos modelos permitirán analizar el comportamiento del muro y sacar conclusiones acerca de la aportación de la geomalla. 


\section{DESCRIPCIÓN DE LA TÉCNICA DE REFUERZO CON GEOMALLA}

El proceso constructivo de viviendas de adobe reforzado con geomallas se recoge en diversas cartillas elaboradas con el fin de difundir la técnica constructiva a raíz del proceso de reconstrucción de las zonas dañadas en el terremoto de Pisco (Perú) de 2007 (27) (28). El refuerzo con mallas sintéticas es considerado también en diversos artículos científicos (24) (25) (26); (29) (30) (31).

La idea fundamental de la técnica de refuerzo con geomalla reside en que el refuerzo envuelva a los muros, trabajando conjuntamente con ellos (Figura 1). Para conseguirlo, la geomalla se une a la mampostería de adobe mediante trozos de cuerda que atraviesan el muro y que son colocadas en las juntas de mortero durante el proceso constructivo de los muros. Este método de unión se complementa mediante un enlucido del muro con mortero de barro en el cual queda embebida la geomalla. La geomalla debe estar además anclada al cimiento en la parte inferior del muro y envolverlo en su parte superior o anclarse a la viga collar que lo corone.

Para que la geomalla trabaje eficientemente envolviendo toda la estructura de adobe se deberán solapar convenientemente los distintos trozos de geomalla que se utilicen, uniéndolos entre sí mediante cordones de cuerda o nylon. Se deberá asegurar que la malla envuelve completamente al adobe, incluyendo los huecos de puertas y ventanas cuando los haya.

En el caso de aplicar la técnica de refuerzo sobre construcciones existentes, como podrían ser construcciones patrimo-

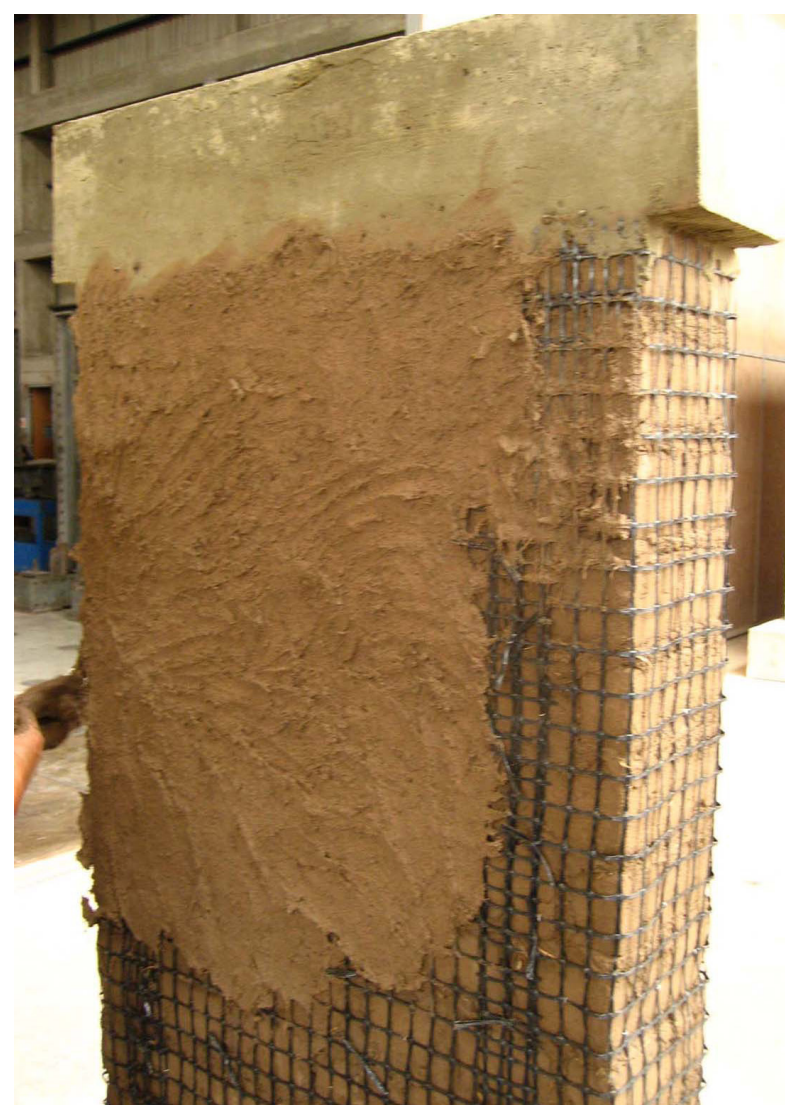

Figura 1. Muro de adobe reforzado con geomalla que ilustra las distintas capas correspondiente a cada fase de construcción (29). niales, la técnica constructiva sería esencialmente similar al proceso antes descrito, si bien en ese caso para la colocación de las cuerdas de unión al muro será necesario realizar algún tipo de taladros en el mismo para poder unir la geomalla al muro en esos puntos.

\section{RESULTADOS EXPERIMENTALES}

\subsection{Caracterización de materiales}

Tanto los ladrillos de adobe como el mortero de unión empleados para la construcción de los muros han sido obtenidos por procedimientos artesanales, empleando una mezcla adecuada de tierra (con contenido suficiente de arcilla), arena, paja y agua. Dada la variabilidad de las propiedades mecánicas de los adobes según las propiedades y proporciones de sus elementos constitutivos, se caracterizó su comportamiento mecánico mediante ensayos de compresión de 5 bloques de adobe apilados (pilas), tal y como se muestra en la Figura 2a. Los bloques de adobe tienen unas dimensiones de $120 \times 210 \times 100 \mathrm{~mm}$. Este ensayo permite determinar la ley de comportamiento de la mampostería de adobe a compresión. Para ello se hace uso de un sensor de desplazamiento en cada cara de la pila para medir las deformaciones del material a partir del desplazamiento relativo entre 2 puntos.

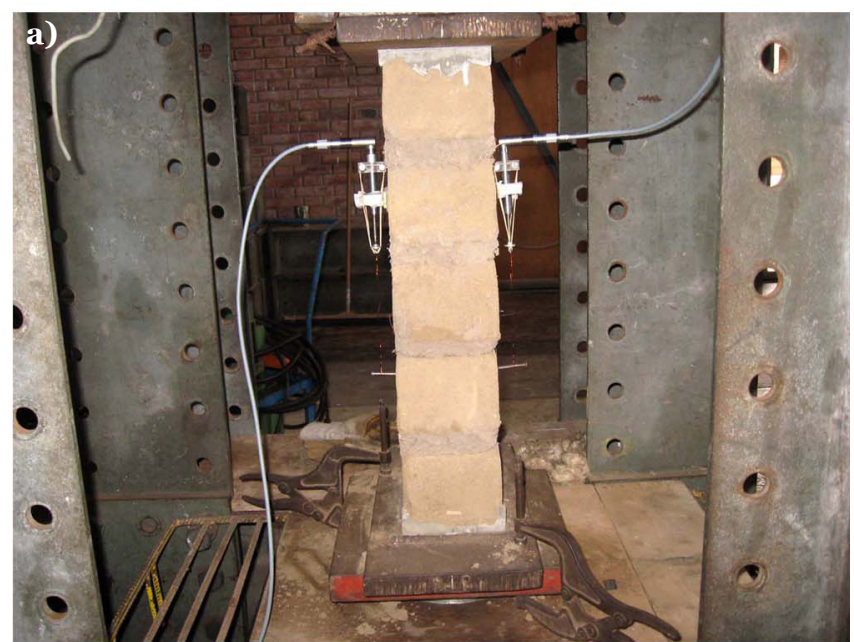

b)

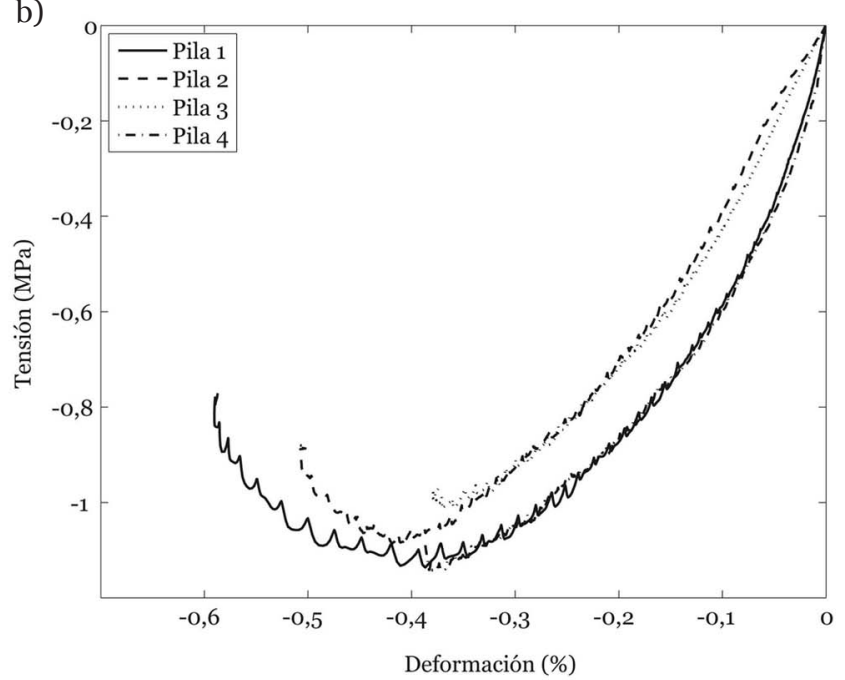

Figura 2. (a) Ensayo de bloques apilados (pilas).

(b) ley de comportamiento obtenida. 
La deformación en la pila se obtiene finalmente haciendo un promedio entre las medidas de las dos caras. La ley de comportamiento obtenida para la mampostería de adobe (pilas) se muestra en la Figura 2b. Se puede apreciar que las cuatro pilas tienen una tensión de comprensión máxima en torno a 1,1 MPa cuando llega a una deformación de compresión del $0,4 \%$.

Para la caracterización del comportamiento de la geomalla se han realizado ensayos de tracción siguiendo las especificaciones de la norma ASTM D6631-01 (Figura 3a). Para la medida de deformaciones se ha empleado un sistema de medición óptico basado en la Correlación Digital de Imágenes. Las dimensiones de los especímenes de geomalla ensayados son de $212 \mathrm{~mm}$ de ancho por $203 \mathrm{~mm}$ de largo. Los resultados obtenidos para cada espécimen ensayado se pueden ver en la Figura $3 b$.

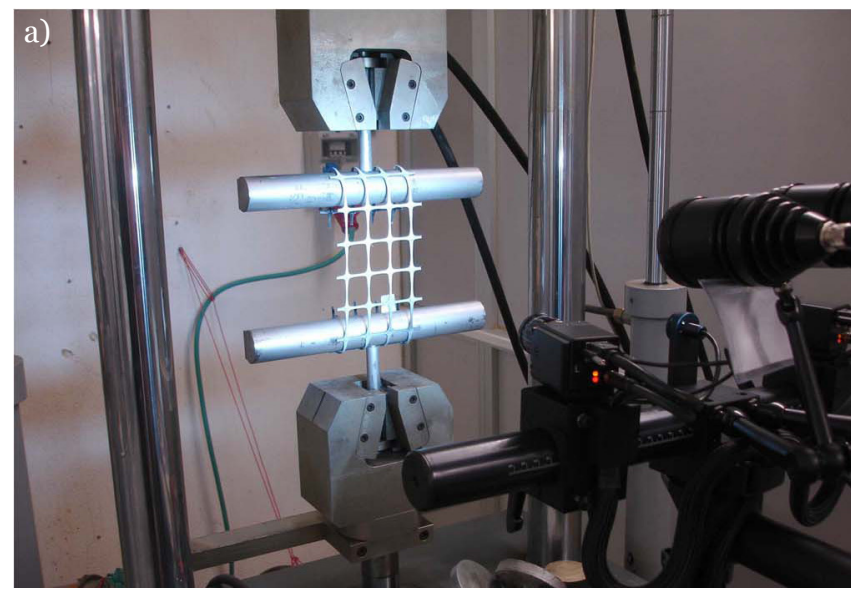

b)

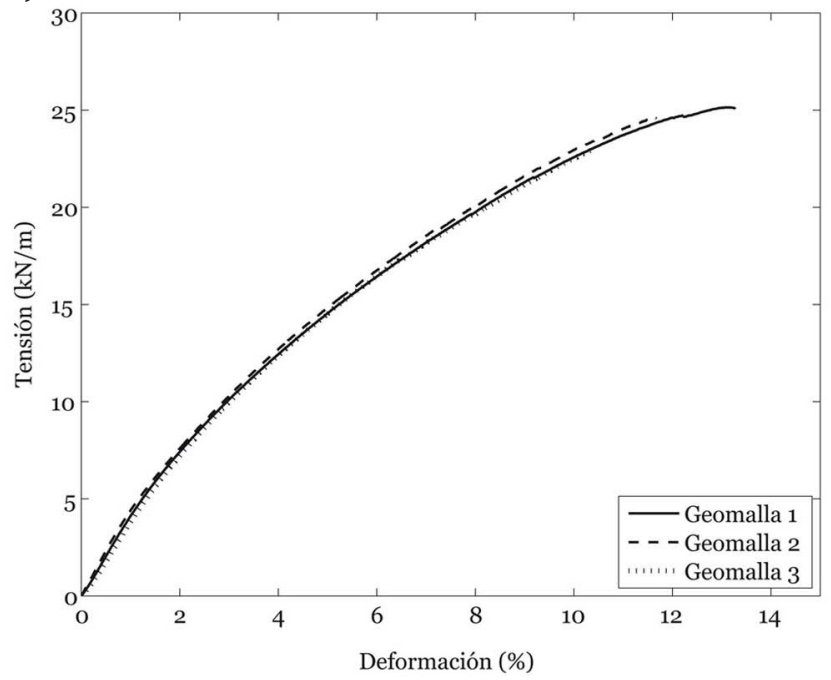

Figura 3. (a) Ensayo a tracción de geomalla.

(b) Ley de comportamiento obtenida.

\subsection{Ensayos de flexión de muros}

Para caracterizar el comportamiento a flexión de los muros se han realizado ensayos de flexión en 3 puntos de muros en posición vertical (Figura 4). Los apoyos articulados superior e inferior del muro se consiguen mediante tiras de neopreno dispuestas en sus secciones extremas, mientras que la carga
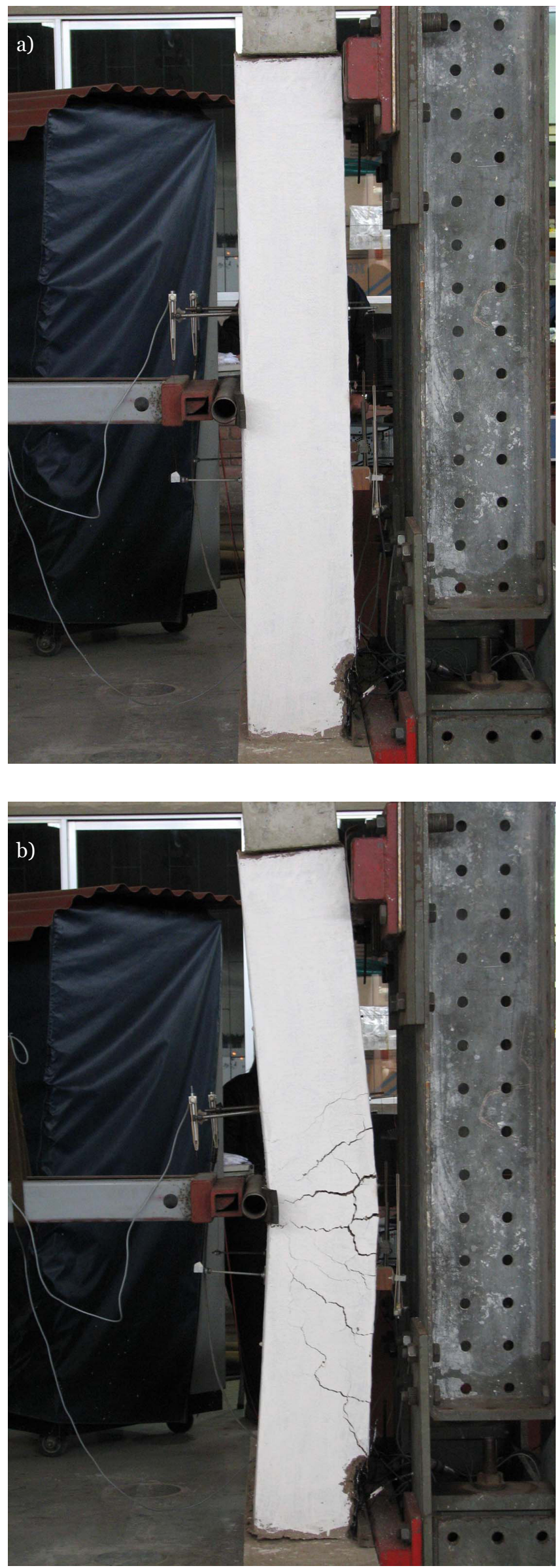

Figura 4. Imágenes del ensayo a flexión.

(a) Antes de aplicar carga. (b) Con carga aplicada. 
del actuador se reparte a lo largo del ancho del muro por medio de un rodillo metálico que transmite la carga al muro a través de otra tira de neopreno. Las dimensiones de los muros fueron de 1,60 $\mathrm{m}$ de alto, o,80 $\mathrm{m}$ de ancho y o,22 $\mathrm{m}$ de espesor. De los tres muros ensayados, uno de ellos no tenía ningún tipo de refuerzo y los otros dos fueron reforzados con geomalla.

En estos ensayos se registró la fuerza aplicada en la sección central del muro, que determina la distribución de momentos flectores (esquema isostático), y simultáneamente se tomaron cuatro medidas de la deformación longitudinal en la sección central del muro: dos de ellas en la cara de tracción $\left(\varepsilon^{+}\right)$y otras dos en una posición equivalente de la cara de compresión del muro $\left(\varepsilon^{-}\right)$. Cada una de estas deformaciones se midió mediante un sensor de desplazamiento que registra el desplazamiento longitudinal relativo entre dos puntos simétricos con respecto a la sección central y separados $40 \mathrm{~cm}$ entre sí. De esta forma, y asumiendo que la flexión del muro cumple la hipótesis de Navier (secciones planas permanecen planas) se tienen dos medidas de la curvatura $(\chi)$ en dicha sección central, obtenida a partir de cada pareja de sensores de tracción y compresión, según la ecuación [1]:

Siendo $h$ el espesor del muro (o,22 m)

$$
\chi=\frac{\varepsilon^{+}-\varepsilon^{-}}{h}
$$

La Figura 5 muestra los resultados conjuntos de la ley momento-curvatura en la sección central de los muros ensayados. Al haber dos medidas de la curvatura para cada muro, se obtienen dos curvas por cada uno de ellos.

Durante los ensayos de los muros reforzados, se realizaron ciclos de carga y descarga, comprobándose el comportamiento dúctil del muro y su capacidad de recuperación del nivel de carga y deformación después de realizar cada ciclo. Se puede observar como todas las curvas experimentales obtenidas son muy similares, por lo que tan solo se considerarán la curva $b$ del muro sin refuerzo y la curva $b$ del muro 2 reforzado para su correlación con los resultados de los modelos analíticos que se presentarán más adelante. En el caso del muro reforzado se tomará además la envolvente de la curva experimental, prescindiendo de los ciclos de carga y descarga. Dichas le-

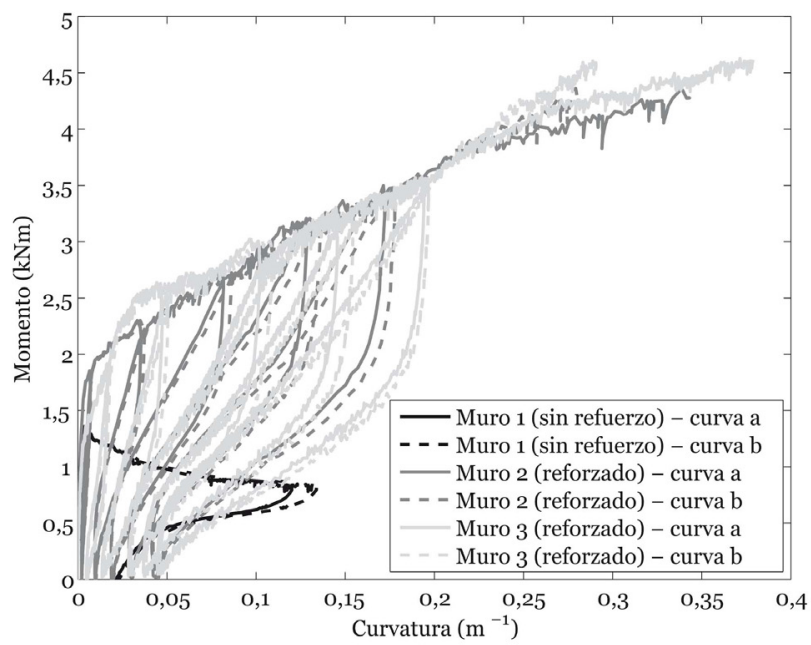

Figura 5. Ley momento curvatura experimental. yes de referencia para el ajuste de los modelos se representan aparte para mayor claridad en la Figura 6.

\section{MODELOS ANALÍTICOS}

\subsection{Ecuaciones que gobiernan el problema}

Las ecuaciones que gobiernan el problema de flexión son ecuaciones de compatibilidad y equilibrio en la sección central del muro. De acuerdo a la hipótesis de Navier, se define a lo largo de la sección transversal del muro (canto $h=0,22 \mathrm{~m}$ en nuestro caso) una distribución lineal de deformaciones. En la Figura 7 se representan las deformaciones a lo largo de la sección, en función de la coordenada , estando la fibra más comprimida en la coordenada $y=0$ (correspondiente a la deformación de la geomalla a compresión $\varepsilon_{g c}$ ) y la fibra más traccionada en $y=h$ (correspondiente a la deformación de la geomalla a tracción $\varepsilon_{q t}$ ). En la coordenada $y=x$ se encuentra la fibra neutra (deformación nula), y el ángulo que forma este perfil de deformaciones con la posición indeformada es la curvatura de la deflexión transversal del muro $(\chi)$. En la representación de la Figura 7 y en las ecuaciones posteriores se ha despreciado la a influencia del enlucido de barro aplicado para recubrir la geomalla.

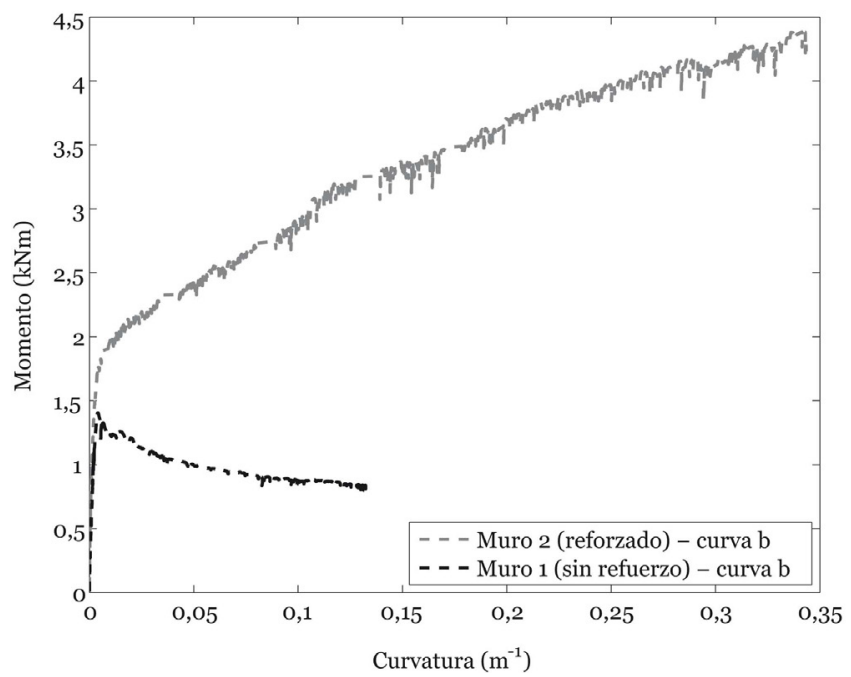

Figura 6. Leyes momento curvatura experimentales de referencia para ajuste de modelos de comportamiento para el muro sin refuerzo y con refuerzo.

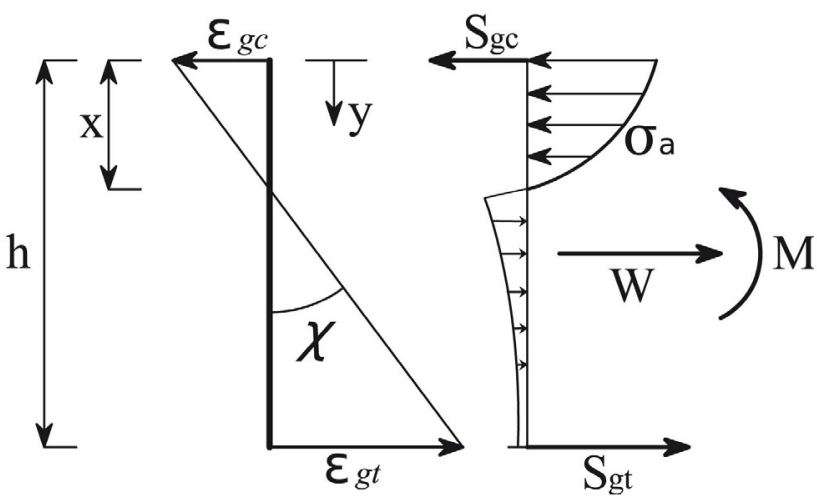

Figura 7. Representación del diagrama de deformaciones y esfuerzos en la sección del muro. 
Las deformaciones de cada punto se pueden escribir en función de la coordenada, de la profundidad de la fibra neutra $x y$ y de la curvatura $\chi$ según la ecuación [2]:

$$
\varepsilon(y, x, \chi)=\chi(y-x)
$$

Las deformaciones de la geomalla a tracción y a compresión serían casos particulares de la ecuación [2] para $y=h$ e $y=0$ respectivamente.

$$
\begin{gathered}
\int_{0}^{h} \sigma_{a}\left(\varepsilon_{a}(y, x, \chi)\right) \cdot b \cdot d y+S_{g c}\left(\varepsilon_{g c}(x, \chi)\right) \cdot b+ \\
+S_{g t}\left(\varepsilon_{g t}(x, \chi)\right) \cdot b=W \\
\int_{0}^{h} \sigma_{a}\left(\varepsilon_{a}(y, x, \chi)\right) \cdot b \cdot(y-h) \cdot d y- \\
-S_{g c}\left(\varepsilon_{g c}(x, \chi)\right) \cdot b \cdot h+W \cdot h / 2=M
\end{gathered}
$$

A partir de la distribución de deformaciones, y conociendo las leyes de comportamiento del material, se puede evaluar el esfuerzo resultante que soporta cada material, debiéndose de cumplirse el equilibrio en la sección. En estas ecuaciones de equilibrio, la resultante del esfuerzo soportado por el adobe viene dado por la integral de las tensiones del material $\left(\sigma_{a}\left(\varepsilon_{a}\right)\right)$ a lo largo de la sección (el ancho del muro viene dado por el parámetro $b=0,8 \mathrm{~m}$ ). Estas tensiones podrán ser tanto de compresión como de tracción, y estarán definidas por la ley de comportamiento del material.

En el equilibrio de fuerzas de la sección -ecuación [3]-, el valor de $W$ es el valor del axil resultante de la carga del peso propio. Dadas las dimensiones de los muros ensayados se ha considerado un valor de $W=-4 \mathrm{kN}$.

Introduciendo la ley de comportamiento de cada material en las ecuaciones de equilibrio, y escribiendo las deformaciones en función de los parámetros $y, x, \chi$ se puede obtener la relación momento-curvatura analíticamente: dando un valor dado a la curvatura $(\chi)$ se puede obtener la profundidad de la fibra neutra $x$ a partir de la ecuación de equilibrio de fuerzas -ecuación [3]-, y sustituyendo en la ecuación de momentos -ecuación [4]-, se obtiene el momento resultante correspondiente al valor de la curvatura impuesto.

\subsection{Modelos de comportamiento y resultados}

La aproximación analítica del comportamiento a flexión se basa en la introducción en las ecuaciones del problema de leyes de comportamiento del material basadas en las obtenidas en los ensayos de caracterización de estos materiales individualmente. Para simplificar los cálculos numéricos estas leyes experimentales son aproximadas mediante tramos lineales. Los valores que definen estas leyes linealizadas se recogen en la Tabla 1, donde cada tramo lineal viene definido por un intervalo de deformación y su correspondiente módulo de elasticidad tangente.

Con el objeto de reproducir aproximadamente el comportamiento real de los muros observado experimentalmente, estas leyes se completan con diversos modelos de comportamiento a tracción del adobe. Se ha supuesto un comportamiento a tracción para el adobe con una ley de ablandamiento progresiva (Figura 8 y Tabla 2) aproximada por tramos lineales en lugar de una rama exponencial (32), de manera que las leyes a tracción quedan definidas también por tramos lineales.

La ley A consiste sencillamente en considerar que el adobe no ofrece ninguna resistencia a la tracción. La ley B supone una rigidez inicial a tracción igual que a compresión (752,5 $\mathrm{MPa})$, hasta alcanzar una tensión máxima a tracción de 0,196 MPa. Esta tensión se corresponde con una estimación de la resistencia a la tracción del adobe obtenida a partir del momento de fisuración del muro sin refuerzo, que se corresponde con el máximo momento obtenido experimentalmente $(1,4 \mathrm{kNm})$. El valor de la tensión de tracción correspondiente se obtiene suponiendo igual rigidez a tracción que a compresión para este momento de fisuración, y sustrayendo el valor de la tensión previa de compresión producida por el peso propio. El valor obtenido permite disponer de un valor de referencia para la resistencia a tracción del adobe. El valor real dependerá de la ley de comportamiento real de adobe a tracción, tanto por su rigidez antes de alcanzar el valor de la tensión máxima como por su curva de ablandamiento.

La ley $\mathrm{C}$ supone una rigidez inicial a tracción menor que el modelo B (369 Mpa) y llega a una tracción máxima también menor al modelo B (o,075 Mpa). Sin embargo el modelo C ofrece una mayor ductilidad que el $\mathrm{B}$, mediante un ablandamiento progresivo hasta llegar a una deformación máxima de 1,5\%.

Tabla 1. Valores de módulo de elasticidad tangente y sus correspondientes intervalos de deformaciones y tensiones de cada tramo linealizado de las leyes de comportamiento experimentales de adobe a compresión y geomalla a tracción.

\begin{tabular}{|c|c|c|c|c|c|}
\hline \multicolumn{2}{|c|}{ Adobe } & \multicolumn{3}{c|}{ Geomalla } \\
\hline $\begin{array}{c}\text { Intervalo } \\
\text { deformación }\end{array}$ & $\begin{array}{c}\text { Módulo tangente } \\
\text { E(MPa) }\end{array}$ & $\begin{array}{c}\text { Intervalo tensión } \\
\text { (MPa) }\end{array}$ & $\begin{array}{c}\text { Intervalo } \\
\text { deformación }\end{array}$ & $\begin{array}{c}\text { Módulo tangente } \\
\text { E(MPa) }\end{array}$ & $\begin{array}{c}\text { Intervalo } \\
\text { tensión (MPa) }\end{array}$ \\
\hline$[0,-0,005)$ & 752,5 & {$[0,-0,376)$} & {$[0,0,02)$} & 374,4 & {$[0,7,48)$} \\
\hline$[-0,005,0-0,0015)$ & 369,0 & {$[-0,376,-0,745)$} & {$[0,02,0,042)$} & 214,1 & {$[7,48,12,19)$} \\
\hline$[-0,0015,-0,0025)$ & 225,9 & {$[-0,745,-0,971)$} & {$[0,042,0,07)$} & 188,8 & {$[12,19,17,48)$} \\
\hline$[-0,0025,-0,004)$ & 99,6 & {$[-0,971,-1,121)$} & {$[0,07,0,106)$} & 142,5 & {$[17,48,22,61)$} \\
\hline$[-0,004,-0,005)$ & $-59,0$ & {$[-1,121,-1,062)$} & {$[0,106,0,1328)$} & 71 & {$[22,61,23,61)$} \\
\hline$[-0,005,-0,0056)$ & $-284,4$ & {$[-1,062,-0,891)$} & - & - & - \\
\hline$[-0,0056,-0,0058)$ & -1649 & {$[-0,891,-0,561)$} & - & - & - \\
\hline$[-0,0058,-0,047)$ & $-0,370$ & {$[-0,561,-0,544)$} & - & - & - \\
\hline
\end{tabular}


Tabla 2. Modelos de comportamiento a tracción del adobe.

\begin{tabular}{|c|c|c|c|c|c|}
\hline \multicolumn{3}{|c|}{ Ley A } & \multicolumn{3}{|c|}{ Ley B } \\
\hline $\begin{array}{l}\text { Intervalo } \\
\text { deformación }\end{array}$ & $\begin{array}{c}\text { Módulo tangente } \\
\text { E(MPa) }\end{array}$ & $\begin{array}{c}\text { Intervalo tensión } \\
\text { (MPa) }\end{array}$ & $\begin{array}{l}\text { Intervalo } \\
\text { deformación }\end{array}$ & $\begin{array}{c}\text { Módulo tangente } \\
\text { E(MPa) }\end{array}$ & $\begin{array}{c}\text { Intervalo tensión } \\
\text { (MPa) }\end{array}$ \\
\hline$[0,-)$ & $\mathrm{o}$ & {$[0,0)$} & {$[0,0,00026)$} & 752,5 & {$[0,0,196)$} \\
\hline- & - & - & {$[0,00026,0,00052)$} & $-752,5$ & {$[0,196,0)$} \\
\hline \multicolumn{3}{|c|}{ Ley C } & \multicolumn{3}{|c|}{ Ley D } \\
\hline $\begin{array}{c}\text { Intervalo } \\
\text { deformación }\end{array}$ & $\begin{array}{c}\text { Módulo tangente } \\
\text { E(MPa) }\end{array}$ & $\begin{array}{l}\text { Intervalo tensión } \\
\text { (MPa) }\end{array}$ & $\begin{array}{c}\text { Intervalo } \\
\text { deformación }\end{array}$ & $\begin{array}{c}\text { Módulo tangente } \\
\text { E(MPa) }\end{array}$ & $\begin{array}{c}\text { Intervalo tensión } \\
\text { (MPa) }\end{array}$ \\
\hline$[0,0,0002)$ & 369 & {$[0,0,075)$} & {$[0,0,0002)$} & 369 & {$[0,0,075)$} \\
\hline \multirow[t]{3}{*}{$0,0002,0,015$} & 5 & {$[0,075,0)$} & {$[0,0002,0,015)$} & 2,70 & {$[0,075,0,035)$} \\
\hline & & & {$[0,015,0,05)$} & o & {$[0,035,0,035)$} \\
\hline & & & {$[0,05,0,05)$} & - & {$[0,035,0)$} \\
\hline
\end{tabular}

Por último, el modelo D se diferencia del C en su ablandamiento, ofreciendo aún mayor ductilidad. Presenta un primer ablandamiento hasta una tensión de 0,035 Mpa y después un comportamiento elasto-plástico perfecto hasta una deformación del $5 \%$.

En todas las leyes propuestas, se asume que la tensión será nula para deformaciones superiores a las indicadas en las tablas correspondientes.

En la Figura 9 se comparan las relaciones obtenidas del momento frente a la curvatura para cada uno de los modelos en el caso de un muro sin refuerzo. Puede observarse que, en el caso de no considerar tracciones en el adobe (modelo A), el muro solo puede soportar momento gracias a la precompresión producida por el peso propio, y su valor máximo $(0,4 \mathrm{KNm})$ es mucho menor que el real. Esto ilustra el hecho de que en construcciones de adobe no reforzadas, es necesario considerar la resistencia a tracción del material para estimar su capacidad máxima de resistencia a flexión, salvo en aquellos casos en los que la carga de peso propio que soporte el muro sea mucho mayor que la resistencia a tracción. Esto puede suceder por ejemplo en construcciones históricas con grandes espesores de muros, algunas de las cuales han soportado sismos de envergadura a lo largo de su historia, constituyendo así ejemplos de cómo se pueden construir edificios sismorresistentes sin necesidad de ningún tipo de refuerzo. Sin embargo, este tipo de diseños basados en grandes espesores de muros no resulta viable con carácter general, por cuestiones económicas y de ordenación urbana. De ahí la necesidad de diseñar sistemas de refuerzo adecuados.

Considerando una ley a tracción como la del tipo B, se observa como la rigidez alcanzada en el tramo inicial de la ley momento-curvatura es excesiva, así como el momento máximo alcanzado, que tiene lugar en el momento en que la fibra más traccionada alcanza su máxima deformación (o,00052). A partir de ese punto el momento cae bruscamente y converge a la curva del modelo sin resistencia a tracción (modelo A). Esto quiere decir que el adobe a tracción presenta presumiblemente una resistencia a tracción menor que la estimada a partir del momento de fisuración del muro, una rigidez menor que la obtenida experimentalmente a compresión y sobre todo una mayor ductilidad.

Los modelos C y D pretenden ilustrar este hecho, y se observa como el modelo $\mathrm{C}$ se adapta en mayor grado al compor- tamiento experimental, dando lugar a un comportamiento algo menos rígido y dúctil que el real. La situación contraria ocurre con el modelo D, que se aleja algo más del comportamiento experimental.

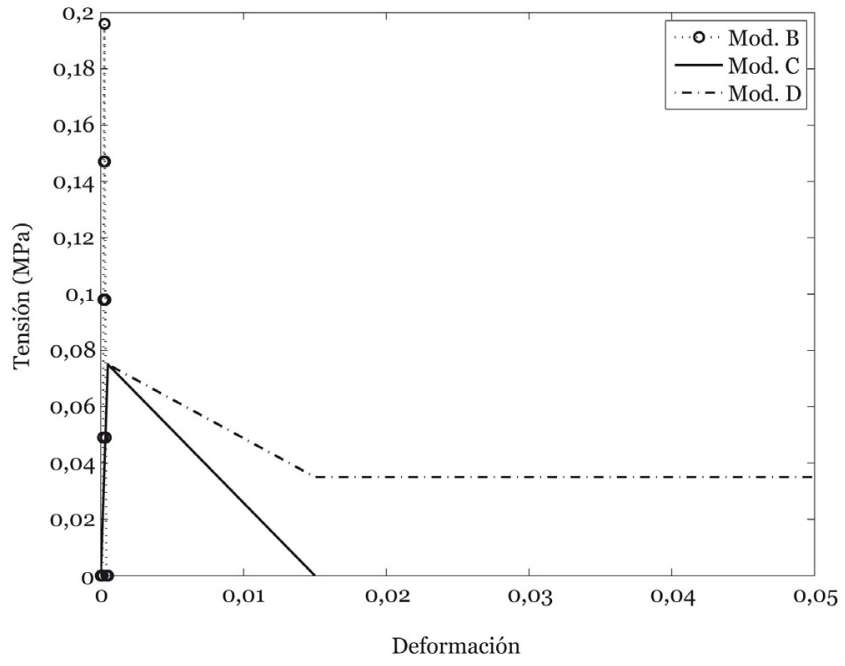

Figura 8. Modelos de comportamiento a tracción del adobe.

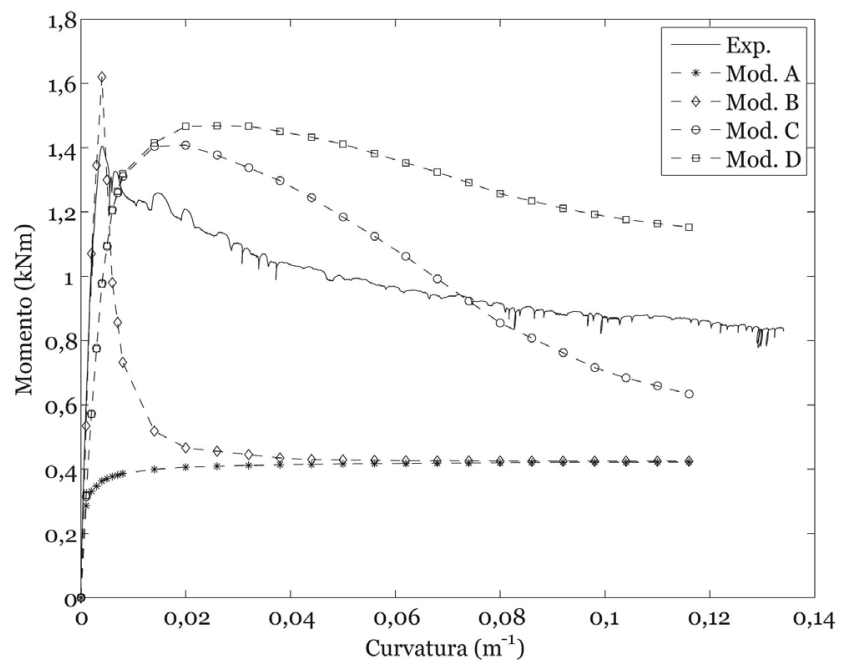

Figura 9. Ley momento curvatura obtenida a partir de modelos. Muro sin reforzar. 
Los resultados obtenidos con estos modelos para el muro reforzado se muestran en la Figura 10. El modelo A se muestra de nuevo incapaz de simular el fenómeno de la fisuración (que tiene lugar aproximadamente para un momento de $1,8 \mathrm{kNm}$ ) y tampoco permite estimar correctamente el valor del momento máximo. Sin embargo, una vez alcanzado el momento de fisuración del modelo A y una vez que el comportamiento a tracción viene definido por la geomalla, se obtiene una pendiente de la gráfica similar a la experimental (rigidez similar), observándose una cierta convergencia hacia el punto de momento máximo obtenido experimentalmente. Esto es debido a que en este tramo la rigidez viene dada fundamentalmente por las geomallas y, en menor medida, por el adobe a compresión, no aportando prácticamente nada la tracción del adobe en la situación próxima a la rotura.

El modelo B ofrece un buen comportamiento hasta el momento de fisuración, pero después la resistencia cae bruscamente y tiende rápidamente al comportamiento tipo A (sin tensiones de tracción en el adobe).

Los modelos C y D se aproximan de nuevo en mayor medida al comportamiento real, observándose sin embargo en este caso mayor nivel de aproximación con el modelo D. Comparando esta situación con la que tenía lugar en el caso del muro sin reforzar, se puede interpretar que la geomalla no solo aumenta por sí misma la resistencia del muro, sino que la cohesión que aporta al adobe contribuye a mejorar sus propiedades, aumentando su resistencia y ductilidad.

Los resultados obtenidos dejan de manifiesto que la relación entre la resistencia a tracción y a compresión de la mampostería de adobe es relativamente alta si se compara con los valores habituales que se dan en otros materiales de construcción, como por ejemplo el hormigón Sin embargo, se puede observar que, considerando una resistencia a compresión aproximada del adobe de $1 \mathrm{MPa}$, y aplicando las correlaciones aproximadas utilizadas habitualmente para deducir la resistencia a tracción del hormigón a partir de su resistencia a compresión (33) (34), se obtendrían valores de la resistencia a tracción del orden de un $30 \%$ de la resistencia a compresión, frente a un valor característico de un 10-15\% para valores habituales de resistencia del hormigón. Esto es debido a que, al igual que ocurre en el hormigón, puede entenderse que a menor resistencia a compresión se tiene una mayor resistencia relativa a la tracción.

Como es lógico, la influencia de este comportamiento a tracción es despreciable a la hora de calcular el momento máximo resistente del muro. Sin embargo, los resultados muestran la alta ductilidad que aporta, comprobándose que resulta imprescindible para obtener una ley aproximada de su comportamiento antes de rotura, que es el que define y caracteriza en definitiva su capacidad de disipar energía y defenderse ante un movimiento sísmico. En caso de no considerar esta resistencia a la tracción (modelo A), la evaluación de la ductilidad no sería correcta.

\section{CONCLUSIONES}

En este artículo se han desarrollado modelos para el comportamiento a flexión de muros de adobe reforzados con geomalla. Si bien los modelos presentados no permiten una aproximación exacta del comportamiento experimental, sin embargo han permitido extraer conclusiones relevantes sobre el comportamiento de la mampostería de adobe con y sin refuerzo de geomalla.

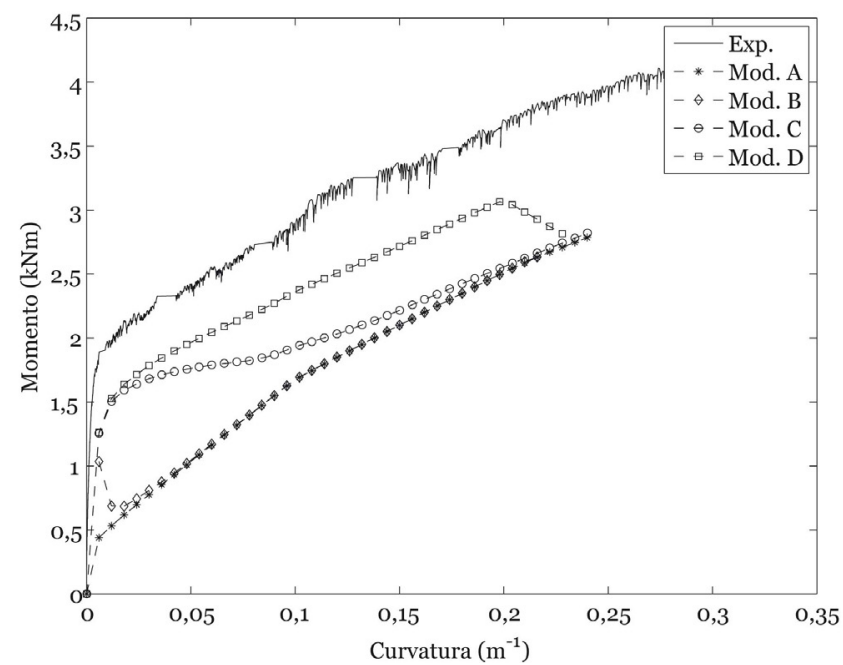

Figura 10. Ley momento curvatura obtenida a partir de modelos. Muro reforzado.

En primer lugar, se ha podido comprobar como la mampostería de adobe ofrece una ductilidad relativamente elevada, a pesar de su baja resistencia a la tracción. Por otro lado, ha quedado demostrada la necesidad de considerar el comportamiento a tracción del adobe a la hora de obtener las leyes de comportamiento a flexión (relación momento-curvatura) de los muros analizados. Estas leyes definen la capacidad de disipación de energía del material ante ciclos de carga y descarga, como son los que ocurren en un terremoto, por lo que resulta necesaria su conocimiento para la evaluación de su capacidad sismorresistente. Sin embargo, como es lógico, esta resistencia a tracción resulta despreciable para el cálculo del momento último de colapso bajo carga estática.

Finalmente, los resultados muestran como la técnica de refuerzo con geomalla constituye una técnica de refuerzo eficaz para construcciones de tierra, aumentando significativamente tanto la resistencia a flexión de los muros como su ductilidad. En el caso concreto de los muros ensayados, el nivel de resistencia alcanzado se multiplicó aproximadamente por 3, mientras que la ductilidad, medida en términos de la curvatura asociada al momento máximo resistente, se multiplicó aproximadamente por 7 .

Los modelos desarrollados y los resultados obtenidos son prometedores y contribuyen a profundizar en el conocimiento de la mampostería de adobe con y sin refuerzo. Sin embargo, deben entenderse como resultados preliminares de cara a futuros trabajos de investigación que permitan profundizar en aspectos tales como la caracterización del comportamiento del adobe a tracción o la influencia del recubrimiento de la geomalla (adherencia y comportamiento a compresión).

\section{AGRADECIMIENTOS}

Los autores agradecen la financiación recibida por parte de la Agencia Española de Cooperación para el Desarrollo (AECID), dentro de su Programa de Cooperación Interuniversitaria, así como a la Consejería de Innovación, Ciencia y Empleo de la Junta de Andalucía a través del Proyecto de Investigación de Excelencia TEP 2068. 


\section{REFERENCIAS}

(1) Dethier, J. (1983). Down to earth: Adobe architecture. New York: Facts On File Inc.

(2) Lynne, E., Adams, C. (2000). Alternative construction: Contemporary natural building methods. New York: Wiley.

(3) Rael, R. (2010). Earth architecture. New York: Princeton Architectural Press.

(4) Jiménez-Delgado, M. C., Cañas-Guerrero, I. (2006). Earth building in Spain. Construction and Building Materials, 20(9): 679-690, doi: http://dx.doi.org/10.1016/j.conbuildmat.2005.02.006.

(5) Bestraten, S., Hormías, E., Altemir, A. (2011). Construcción con tierra en el siglo XXI. Informes de la Construcción, 63(523): 5-20, doi: http://dx.doi.org/10.3989/ic.10.046.

(6) UNESCO. (2003). http://whc.unesco.org/en/wallmap.

(7) Alva, A. (2001). The conservation of earthen architecture. The Getty Conservation Institute Newsletter, 16(1): 4-11.

(8) Spence, R. (2007). Saving lives in earthquakes: Successes and failures in seismic protection since 1960. Bulletin of Earthquake Engineering, 5(2): 139-251, doi: http://dx.doi.org/10.1007/s10518-006-9028-8.

(9) Razani, R. (1978). Seismic protection of unreinforced masonry and adobe low-cost housing in less developed countries: Policy issues and design criteria. Disasters, 2(2-3): 137-147, doi: http://dx.doi.org/10.1111/j.1467-7717.1978.tbooo82.x.

(10) Morel, J. C., Pkla, A., Walker, P. (2007). Compressive strength testing of compressed earth blocks. Construction and Building Materials, 21(2): 303-309, doi: http://dx.doi.org/10.1016/j.conbuildmat.2005.08.021.

(11) Guillaud, H. (2001). Characterization of earthen materials. En Avrami, E., Guillaud, H., Hardy, M. (Eds.) Terra Literature Review: An Overview of Research in Earthen Architecture Conservation (pp. 21-31). Los Angeles: The Getty Conservation Institute.

(12) Piattoni, Q., Quagliarini, E., Lenci, S. (2011). Experimental analysis and modelling of the mechanical behaviour of earthen bricks. Construction and Building Materials, 25(4): 2067-2075, doi: http://dx.doi.org/10.1016/j.conbuildmat.2010.11.039.

(13) Varum, H., Figueiredo, A., Silveira, D., Martins, T., Costa, A. (2011). Investigaciones realizadas en la universidad de Aveiro sobre caracterización mecánica de las construcciones existentes en adobe en Portugal y propuestas de rehabilitación y refuerzo. Resultados alcanzados. Informes de la Construcción, 63(523): 127-142, doi: http://dx.doi.org/10.3989/ ic.10.016.

(14) Silveira, D., Varum, H., Costa, A., Martins, T., Pereira, H., Almeida, J. (2012). Mechanical properties of adobe bricks in ancient constructions. Construction and Building Materials, 28(1): 36-44, doi: http://dx.doi.org/10.1016/j.conbuildmat.2011.08.046.

(15) Construcciones, R. N. d. (2000). Norma Técnica de Edificación NTE o.8O ADOBE (Adobe Building Standard, in spanish). Lima: SENCICO.

(16) Minke, G. (2001). Construction manual for earthquake-resistant houses built of earth. Eschborn: Gate-Basin at GTZ $\mathrm{GmbH}$.

(17) IAEE. (1986). Guidelines for earthquake-resistant non-engineered construction. International Association for Earthquake Engineering.

(18) Cao, Z., Watanabe, H. (2004). Earthquake response predication and retrofitting techniques of adobe structures. En Proceedings of the 13th World Conference on Earthquake Engineering. Vancouver (Canada).

(19) Mayorca, P., Meguroe, K. (2008). A step towards the formulation of a simple method to design pp-band mesh retrofitting for adobe masonry houses. En Proceedings of the 14th World Conference on Earthquake Engineering. Beijing (China).

(20) Rojas-Brabo, J., Vendouw, W., Bijvoet, M. (2004). Improvement of the earthquake resistance of existing adobe dwellings in Cuzco, Peru. En Proceedings of the 13th World Conference on Earthquake Engineering. Vancouver (Canada).

(21) Fonseca-Ferreira, C., D’Ayala, D. (2012). Seismic assessment and retrofitting of peruvian earthen churches by means of numerical modelling. En 15th World Conference Earthquake Engineering. Lisboa (Portugal).

(22) Tarque, N., Camata, G., Spacone, E., Varum, H., Blondet, M. (2010). Numerical modelling of in-plane behaviour of adobe walls. En Sísmica 2010, 8th Congresso de Sismología e Engenharia Sismica. Aveiro (Portugal).

(23) Jayasinghe, C., Mallawaarachchi, R. S. (2009). Flexural strength of compressed stabilized earth masonry materials. Materials and Design, 30(9): 3859-3868, doi: http://dx.doi.org/10.1016/j.matdes.2009.01.029.

(24) Blondet, M., Vargas, J., Tarque, N., Iwaki, C. (2011). Construcción sismorresistente en tierra: La gran experiencia contemporánea de la Pontificia Universidad Católica del Perú. Informes de la Construcción, 63(523): 41-50, doi: http:// dx.doi.org/10.3989/ic.10.017.

(25) Blondet, M., Torrealva, D., Vargas, J., Velasquez, J.y Tarque, N. (2006). Seismic reinforcement of adobe houses using external polymer mesh. En Proceedings of First European Conference on Earthquake Engineering and Seismology. Geneva (Switzerland).

(26) Torrealva, D., Cerrón, C., Espinoza, Y. (2008). Shear and out of plane bending strength of adobe walls externally reinforced with polypropylene grids. En Proceedings of the 14th World Conference on Earthquake Engineering. Beijing (China).

(27) Vargas, J., Torrealva, D., Blondet, M. (2007). Construcción de viviendas seguras y saludables. Lima, Peru: Pontificia Universidad Catolica del Peru (PUCP).

(28) GTZ. (2007). Construction manual for earthquake-resistant houses with adobe. Geogrid technology. Lima, Peru: COSUDE and GTZ - Swiss and German Offices for Development Cooperation Office.

(29) Blondet, M., Vargas, J., Patron, P., Stanojevich, M., Rubiños, A. (2008). A human development aprroach for the construction of safe and and healthy houses in seismic areas. En Proceedings of 14th World Conference on Earthquake Engineering. Beijing (China). 
(30) Figueiredo, A., Varum, H., Costa, A., Silveira, D., Oliveira, C. (2013). Seismic retrofitting solution of an adobe masonry wall. Materials and Structures, 46(1-2): 203-219, doi: http://dx.doi.org/10.1617/s11527-012-9895-1.

(31) Noguez, R., Navarro, S. (2005). Reparacion de muros de adobe con el uso de mallas sinteticas. En International Conference SismoAdobe2005. Lima (Peru)

(32) Karihaloo, B. L. (1995). Fracture mechanics and structural concrete. London: Longman.

(33) J. Calavera, L. G. (1992). Cálculo de flechas en estructuras de hormigón armado. Madrid: Intemac.

(34) Ministerio de Fomento. (2008). EHE-o8: Instrucción de hormigón estructural. Madrid: Ministerio de Fomento. 